\title{
Machine vision-based cocoa beans fermentation degree assessment
}

\author{
YRO Aubain ${ }^{1 *}$, N’ZI Édié Camille ${ }^{1}$, KPALMA Kidiyo ${ }^{2}$ \\ ${ }^{1}$ Institut National Polytechnique Félix Houphouët-Boigny, Yamoussoukro, Côte d’Ivoire \\ ${ }^{2}$ Univ Rennes, INSA Rennes, CNRS, IETR - UMR 6164, F-35000 Rennes, France \\ *aubainyro@gmail.com
}

\begin{abstract}
Fermentation degree is one of the main important indicators of cocoa bean quality. Therefore, accurate estimation of fermentation degree is very important for ensuring the quality of final products. This paper presents a quantitative method for assessing the cocoa beans fermentation degree by image analysis. In this approach, the image of cocoa beans are acquired using a camera and processed to obtain the bean's target. Then, the target's pixels are clustered into red, green and blue regions where each region's pixel presents respectively a maximal value of R, G and B in RGB color space. After that, the first three color moments of each region are calculated from RGB space and used to describe the fermentation degree of the bean. Finally, multi-class support vector machine (SVM) algorithm is used as classifier to discriminate cocoa beans sample into unfermented (UF), partly fermented (PF) and well fermented (WF) categories. Experimental results show that $99.17 \%$ of UF beans, $97.50 \%$ of PF beans and $100 \%$ of WF beans were detected successfully. This results revealed that the proposed method could be used as a fast, accurate and a reliable tool to discriminate cocoa beans according to their fermentation degree for quality assurance.
\end{abstract}

Keywords: Cocoa bean, Fermentation degree, Image analysis, Machine vision, SVM.

\section{Introduction}

Cocoa beans are the seeds from fruit pods of the tree Theobroma L. [1]. They are well known as the main raw material of chocolate and of a vast range of products like cocoa powder, cocoa beverages, bakery products and ice cream [2].

Worldwide cocoa is an important agricultural crop and it is one of the most exported raw material. However, before cocoa beans can be traded and processed into final industrial they have to undergo post-harvest processing on farms and plantations [3][5]. The fermentation is the critical post-harvest step to improve the quality of the beans [6]-[8]. Indeed, the flavour and aroma precursors which reduces the astringency and bitterness of the beans are produced during fermentation [3]. Therefore, the fermentation degree of cocoa beans is an important quality indicator which determine the acceptability or rejection of beans on the international market [3], [5], [6], [9]. 
Traditionally, cut-test is used to assess fermentation degree of cocoa beans [3], [9], [10]. It is a simple visual method which involves cutting a number of beans lengthwise and analyzing their internal color [4]: unfermented beans are slaty, partly fermented beans are purple or violet and fully or well fermented beans are brown in color. However, this method is time-consuming, subjective and not reliable.

This paper proposed a simple color image analysis method for assessing cocoa fermentation degree by using RGB color features and a multi-class support vector machine algorithm.

\section{$2 \quad$ Materials and methods}

\subsection{Cocoa beans sample}

The cocoa beans samples used in this study were acquired by the National Polytechnic Institute of Yamoussoukro in Côte d'Ivoire (7 32' 23.96" N; 5 32' 49.488" W).

A total of 600 beans samples are manually selected by a quality control expert and image of each bean was captured by a computer vision system. The cocoa beans were divided into three classes of fermentation degree: unfermented (Class 1), partly fermented (Class 2) and well fermented (Class 3) were identified in this research. The training set consists of 80 images per class forming a set of 240 images and the remaining images which consists of 120 images for each class were used as testing set.

\subsection{Image acquisition}

Figure 1 shows the acquisition system developed in the laboratory. For image acquisition, a color coupled charge device (CCD) camera (SONY XCG-5005CR, Japan) with lens zoom 16 mm (Fujifilm corporation, model HF16HA-1B, Japan) was used. The image acquisition card (Mil Matrox) was used for transferring information from camera to computer (Core-i7 CPU: $2.5 \mathrm{GHz}$; RAM: $4 \mathrm{~GB}$ ). To ensure a correct and consistent lighting throughout the acquisition process, two white LED with 18 watts were used. Once the camera has been set, images have been acquired in tiff format with $512 \times 512$ pixels resolution.

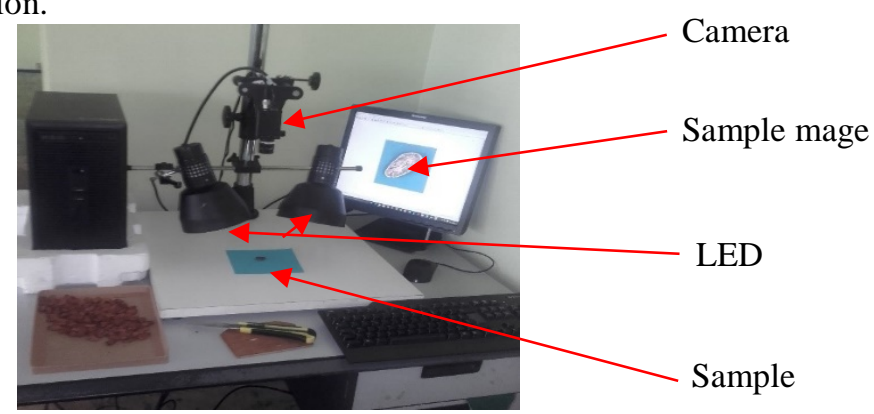

Fig. 1. The cocoa bean image acquisition system. 


\subsection{Image Preprocessing}

This image preprocessing step aims to identify each cocoa bean target from the image background.

Firstly, each image were resized to 256x256 pixels in order to reduce images processing time and then they were segmented. To isolate the target from the background, the b gray image of CIELAB color space were segmented using Otsu's thresholding method. After that, median blur type smoothing, morphological opening and closing were used to remove single noise pixels. Finally, the binary image was used as masking on the input image to obtain the desired image of cocoa beans. The preprocessing steps performed on the cocoa beans samples are shown in Fig. 2.

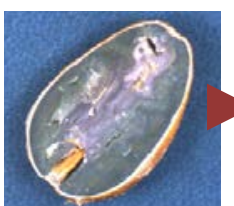

(a)

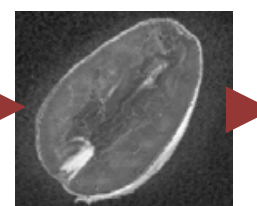

(b)

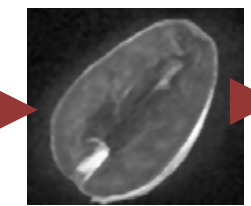

(c)

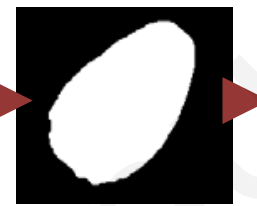

(d)

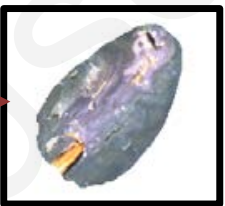

(e)

Fig. 2. The chart of processing steps for segmenting the cocoa beans image from a noisy background: (a) the raw beans sample image; (b) gray image of channel b of the Lab image; (c) after the noise cancellation process; (d) binary image after morphological opening and closing and filling process; and (e) after the segmentation of the beans image from the background.

\section{$2.4 \quad$ Features extraction}

After the desired cocoa beans image was isolated from the background, the mean, the standard deviation and the skewness were extracted using RGB color space. A total of 27 color feature were extracted to describe each cocoa bean target.

In order to obtain this color features, we identified three color regions of each bean surface. The color region namely red, green and blue regions are identified as the region in which each pixel presents the maximal value of $R, G$ and $B$ channels in the RGB color space, respectively. Let $P_{i}=(R, G, B)$ be the ith pixel of the cocoa bean, with its three colors components in the RGB color space. If the maximal value of the three colors components is $R$, the pixel $P_{i}=(R, G, B)$ is classified as the pixel of the red region of cocoa bean. For example, the red region is determined according to equation (1):

$$
C_{R}=\left\{P_{i}=(R, G, B) / R>\max (G, B), i=1, \ldots, N\right\}
$$

Were $C_{R}$ is the set of the pixels in the red region and $\mathrm{N}$ is the total number of the pixel which represents the cocoa bean target in the image. 


\subsection{Classification}

Finally, a Support Vector Machine (SVM) algorithm was attempted to develop discrimination model which were applied to classify the cocoa beans in three categories fermentation degree: unfermented, partly fermented and well fermented.

SVM is a nonlinear supervised learning method for classification and regression problems which was developed by Vapnik. The basic purpose of an SVM algorithm is to obtain the optimal boundary which separates the different classes in the training set. The optimal performance of SVM is dependent on the choice of the kernel function. For this work, Gaussian radial basis function (RBF) is chosen as the kernel function.

\section{$3 \quad$ Results and discussion}

In this study, we used the confusion matrix and the classification accuracy to evaluate the performance of the proposed method.

Experimental results obtained using the proposed method is shown in Table 1. It can be seen, this algorithm could distinguish between unfermented, partly fermented and well fermented beans with $99.17 \%$, 97.50\% and 100\% respectively. The highest error of classification was related to class 2 (partly fermented beans) with $97.50 \%$. For this class, 1 bean was wrongly allocated into class 1 (unfermented beans) and 2 others beans were allocated wrongly into class 3 (well fermented beans). However, the beans samples in class 3 (well fermented beans) are successfully classified.

Misclassification of the 1 partly fermented bean as the unfermented bean and the 2 partly fermented beans to well fermented beans class was related to the color properties overlap between the three classes.

The rate of well-classified images obtained by this proposed approach using color features in RGB color space was $98.89 \%$. Among 360 samples, only 4 beans sample were wrongly classified. This means that this classifier system had the lowest error and an appropriate accuracy.

Table 1. Classification performances of the proposed method.

\begin{tabular}{lccccc}
\cline { 2 - 6 } & \multicolumn{3}{c}{ Predicted classes } & & Performance \\
\cline { 2 - 6 } & Class & Class 2 & Class 3 & Num. beans & $\begin{array}{c}\text { Accuracy } \\
(\%)\end{array}$ \\
\hline Class 1 & 1 & 1 & 0 & 120 & 99,17 \\
Class 2 & 1 & 117 & 2 & 120 & 97,50 \\
Class 3 & 0 & 0 & 120 & 120 & 100 \\
Total accuracy & & & & & 98,89 \\
\hline
\end{tabular}




\section{Conclusion}

In this work we proposed an automatic procedure for estimating the cocoa beans fermentation degree. Based on computer vision methods, this system is easy to use and gives repeatable results of images classification. The classification accuracy obtained using color feature for unfermented, partly fermented and well fermented cocoa beans was $98.89 \%$. These results shows that the proposed system can achieve very high classification performance. Finally, this study have proven that the presented system can potentially be used as a routine analytical tool for cocoa beans fermentation degree assessment. In the future we plan to increase the number of images per training class and to investigate combining color and texture features for cocoa beans fermentation degree assessment process by machine vision.

\section{Acknowledgements}

This work is supported by the Strategic Support Program for Scientific Research (PASRES-CI) of Côte d'Ivoire. The first authors gratefully acknowledge IETR/INSARennes laboratory for its valuable collaboration.

\section{References}

[1] E. Teye, X. Huang, F. Han, et F. Botchway, « Discrimination of cocoa beans according to geographical origin by electronic tongue and multivariate algorithms ", Food Anal. Methods, vol. 7, n 2, p. 360-365, 2014.

[2] M. S. Beg, S. Ahmad, K. Jan, et K. Bashir, «Status, supply chain and processing of cocoa - A review », Trends Food Sci. Technol., vol. 66, p. 108-116, août 2017.

[3] P. Aculey et P. Snitkjaer, "Ghanaian cocoa bean fermentation characterized by spectroscopic and chromatographic methods and chemometrics ", Journal of Food Science, p. 300-307, 2010.

[4] N. León-Roque, M. Abderrahim, L. Nuñez-Alejos, S. M. Arribas, et L. Condezo-Hoyos, « Prediction of fermentation index of cocoa beans (Theobroma cacao L.) based on color measurement and artificial neural networks », Talanta, vol. 161, p. 31-39, déc. 2016.

[5] O. A. Emmanuel, Q. Jennifer, S. B. Agnes, S. T. Jemmy, et K. S. Firibu, «Influence of pulp-preconditioning and fermentation on fermentative quality and appearance of Ghanaian cocoa (Theobroma cacao) beans. ", Int. Food Res. J., vol. 19, nº 1, 2012.

[6] E. Teye et al., « Estimating cocoa bean parameters by FT-NIRS and chemometrics analysis », Food Chem., vol. 176, p. 403-410, 2015.

[7] G. G. D. Diomande, « Contribution des méthodes spectroscopiques et isotopiques à la caractérisation géographique et phénotypique du cacao », 2014.

[8] L. Bankoff, G. H. Ouattara, T. G. Karou, S. T. Guehi, J. G. Nemlin, et J. K. Diopoh, "Impacts de la fermentation du cacao sur la croissance de la flore microbienne et la qualite des feves marchandes ", Agron. Afr., vol. 25, n 2, p. 159-170, 2013.

[9] J. E. Kongor, J. F. Takrama, A. S. Budu, H. Mensah-Brown, et E. O. Afoakwa, « Effects of fermentation and drying on the fermentation index and cut test of pulp preconditioned Ghanaian cocoa (Theobroma cacao) beans », J. Food Sci. Eng., vol. 3, n ${ }^{0}$ 11, p. 625, 2013.

[10] ISO, « Fèves de cacao - Épreuve à la coupe (ISO 1114) ». Genève: Organisation internationale de normalisation, 1977. 